\section{Marcadores para o diagnóstico e tratamento de 924 gestações com diabetes melito gestacional}

\author{
Markers of diagnosis and treament in 924 pregnancies \\ with gestational diabetes mellitus
}

Josiane Cristine Melchioretto Detsch', Ana Cristina Ravazzani de Almeida', Luis Gustavo Cambrussi Bortolini', Denis José Nascimento², Fernando Cesar Oliveira Junior'², Rosângela Roginski Réa'

\section{RESUMO}

Objetivos: Avaliar o perfil epidemiológico e a evolução de mulheres com diabetes melito gestacional (DMG), determinando fatores de risco para maior vigilância. Sujeitos e métodos: Foram estudadas 924 gestações de 916 pacientes, de 6 de novembro de 2001 a 21 de setembro de 2009. Resultados: Foram encontrados fatores de risco para DMG em 95,1\% dos casos. A prevalência de diabetes materno, paterno e em outros familiares foi de $24,3 \%, 9,4 \%$ e $24,7 \%$, respectivamente. Os fatores preditivos para uso de insulina foram: glicemia de jejum (GJ) no rastreamento $\geq 85$, GJ no Teste Oral de Tolerância à Glicose (TOTG) $\geq 95$, glicemia 2 h após 75 $\mathrm{g}$ de glicose $\geq 200 \mathrm{mg} / \mathrm{dL}$, DMG prévio, obesidade, $\mathrm{HbA1c}>6 \%$ e história familiar de DM em parente de primeiro grau associada à obesidade ou DMG prévio, esta última a associação mais relevante $(p<0,05)$. Conclusões: Os fatores de risco analisados se mostraram altamente sensíveis para a detecção de DMG, e a disposição da história familiar reforça sua relação com o DM2. Recomenda-se maior vigilância a gestantes com fatores preditivos para necessidade de insulina. Arq Bras Endocrinol Metab. 2011;55(6):389-98

\section{Descritores}

Diabetes gestacional; fatores de risco; controle metabólico; tratamento; insulina; complicações

\begin{abstract}
Objectives: To evaluate the epidemiological profile and outcomes of women with gestational diabetes mellitus (GDM), determining risk factors for increased vigilance. Subjects and methods: We studied 924 pregnancies in 916 patients between November 6, 2001 and September 21, 2009. Results: Risk factors were found in $95.1 \%$ of cases. The prevalence of maternal diabetes, paternal diabetes and diabetes in other family members was $24.3 \%, 9.4 \%$ and $24.7 \%$, respectively. Predictive factors for insulin use were: screening fasting glucose (FG) $\geq 85$, Oral Glucose Tolerance Test (OGTT) FG $\geq 95$, $2 \mathrm{~h}$ glucose after $75 \mathrm{~g} \geq 200 \mathrm{mg} / \mathrm{dL}$, previous GDM, obesity, $\mathrm{HbA} 1 \mathrm{c}>6 \%$, and the association of risk factors including family history of diabetes mellitus and obesity or previous GDM, the last one the most relevant $(p<0,05)$. Conclusions: Risk factors were very sensitive for GDM detection, and provision of family history strengthens its relationship with T2DM. Greater vigilance is recommended for patients with predictive factors for insulin requirement. Arq Bras Endocrinol Metab. 2011;55(6):389-98
\end{abstract}

Keywords

Gestational diabetes; risk factors; metabolic profile; treatment; insulin; complications
'Serviço de Endocrinologia, Hospital de Clínicas, Universidade Federal do Paraná (SEMPR), Curitiba, PR, Brasil

${ }^{2}$ Setor de Ginecologia e Obstetrícia, Hospital de Clínicas, Universidade Federal do Paraná UFPR, Curitiba, PR, Brasil
Rosângela Roginski Réa Serviço de Endocrinologia e Metabologia, Hospital de Clínicas, Universidade Federal do Paraná Av. Agostinho Leão Junior, 285 80030-110 - Curitiba, PR, Brasil rosangelarea@uol.com.br

Recebido em 4/Out/2010 Aceito em 21/Jul/2011

\section{INTRODUÇÃO}

$\mathrm{D}$ iabetes melito gestacional (DMG) é definido como qualquer grau de intolerância à glicose com início ou primeiro reconhecimento durante a gestação, poden- do ou não persistir após o parto $(1,2)$. Sua prevalência é de $7 \%$ de todas as gestações, podendo variar de $1 \%$ a $14 \%$ dependendo da população e resultando em 200 mil casos por ano nos Estados Unidos (3). No Brasil, segundo da- 
dos levantados pelo Segundo Estudo Brasileiro de Diabetes Gestacional, a prevalência é de 7,6\% (4).

Muitos estudos têm avaliado a presença e ausência de indicadores de risco para o diabetes gestacional e a eventual capacidade para identificar pacientes cuja evolução não justificaria um cuidado especial. Os principais fatores reconhecidos até o momento são: história familiar de diabetes em familiar de primeiro grau, obesidade, idade superior a 25 anos, hipertensão arterial sistêmica, mau passado obstétrico (antecedentes obstétricos de morte fetal ou neonatal, história de macrossomia fetal ou diabetes gestacional prévios, abortos de repetição e malformações congênitas fetais) e macrossomia, polidrâmnio, doença hipertensiva específica da gestação (DHEG) ou ganho de peso excessivo na gestação em curso (5). Mais recentemente, a baixa estatura da gestante $(\leq 151 \mathrm{~cm})$ e a síndrome dos ovários policísticos (SOP) também foram descritas como fatores de risco para DMG $(6,7)$.

A Organização Mundial da Saúde (OMS) e a Sociedade Brasileira de Diabetes (SBD) recomendam o rastreamento de todas as gestantes para diabetes gestacional, independentemente da presença de fatores de risco, com realização de glicemia de jejum na primeira consulta do pré-natal, por ser um exame simples e de baixo custo $(8,9)$. Dessa forma, reconhecem a importância da gestação como o período, frequentemente único, no qual mulheres que vivem em países em desenvolvimento irão procurar atendimento médico e realizar exames de rotina. $\mathrm{O}$ ponto de corte da glicemia de jejum para o rastreamento positivo, independente do momento da gravidez, foi estabelecido em $85 \mathrm{mg} /$ dL $(8,9)$. Um resultado inferior a $85 \mathrm{mg} / \mathrm{dL}$ é então considerado rastreamento negativo e, na ausência de fatores de risco para $\mathrm{DMG}$, o rastreamento não seria repetido durante o restante da gestação.

Já o procedimento diagnóstico recomendado pela OMS e pela American Diabetes Association (ADA) é o teste de sobrecarga com $75 \mathrm{~g}$ de glicose $(10,11)$, que deve ser realizado entre 24 e 28 semanas de gestação. Porém, se o rastreamento for positivo no primeiro trimestre, ou a gestante apresentar vários fatores de risco, este teste pode ser realizado mais precocemente. Na época da realização desse estudo, eram recomendados para o diagnóstico do diabetes gestacional os pontos de corte de $110 \mathrm{mg} / \mathrm{dL}$ para a glicemia de jejum e de $140 \mathrm{mg} / \mathrm{dL}$ para o valor de 2 horas após sobrecarga com $75 \mathrm{~g}$ de glicose (9).

É reconhecido que o DMG implica aumento do risco de complicações durante a gestação, tanto para a mãe como para o feto (12). A gestante com DMG não tratada tem maior risco de ruptura prematura de membranas, parto pré-termo, feto macrossômico, e maior incidência de pré-eclâmpsia. Além disso, o DMG representa um aumento no risco de diabetes tipo 2 (DM2) posterior à gestação $(8,9)$, devendo ser utilizado como instrumento na sua prevenção, ou seja, com base no Diabetes Prevention Program (DPP) devem ser recomendados a essas pacientes no pós-parto um controle dietoterápico e a realização de atividades físicas, como forma de reduzir ou retardar a progressão para o DM2 (13).

O objetivo deste estudo foi determinar o perfil epidemiológico das gestantes com DMG acompanhadas no Ambulatório de Pré-Natal de Pacientes com Diabetes Gestacional e Pré-Gestacional do Hospital de Clínicas da Universidade Federal do Paraná, no período compreendido entre 6 de novembro de 2001 a 21 de setembro de 2009. Além disso, correlacionar esses dados com a evolução das pacientes durante a gestação, incluindo o controle glicêmico, tratamento e complicações obstétricas e, por fim, determinar a presença de grupos para os quais se faria necessária maior vigilância durante a gestação, ou para os quais esta poderia ser dispensável.

\section{SUJEITOS E MÉTODOS}

Foram objeto deste estudo pacientes encaminhadas ao Ambulatório de Diabetes do Pré-Natal do Serviço de Endocrinologia, do Hospital de Clínicas da Universidade Federal do Paraná, com valores glicêmicos alterados, com ou sem fatores de risco identificados para DMG, e sem diagnóstico de diabetes melito anterior à gestação (diabetes tipo 1 ou tipo 2).

A amostra foi constituída de 916 pacientes (924 gestações) que foram acompanhadas no período de 6 de novembro de 2001 a 21 de setembro de 2009.

Durante a primeira consulta foi preenchido um questionário padronizado com questões referentes à sua história médica pregressa, peso pré-gestacional, história familiar de diabetes, antecedentes médicos e obstétricos e informações relacionadas à gestação em curso. A altura da paciente e seu peso na época em que o diagnóstico de DMG foi feito, por meio do Teste Oral de Tolerância à Glicose (TOTG), foram obtidos a partir do prontuário das pacientes provenientes da própria Instituição ou da Carteira de Pré-Natal das demais pacientes. Foram estudados neste trabalho os seguintes fatores de risco para DMG: histórico de diabetes em 
familiares, idade superior a 25 anos, obesidade (índice de massa corporal [IMC] pré-gestacional $\geq 30 \mathrm{~kg} / \mathrm{m}^{2}$ ), altura $\leq 1,51 \mathrm{~m}$, hipertensão arterial sistêmica crônica, mau passado obstétrico (polidrâmnio, macrossomia e história de abortos de repetição ou óbito fetal prévio), doença hipertensiva específica da gestação (DHEG) ou ganho de peso excessivo (superior a $500 \mathrm{~g} /$ semana) na gestação estudada e polidrâmnio ou crescimento fetal excessivo quando constatados previamente ao diagnóstico de DMG.

Para avaliação do controle glicêmico, foram realizados "perfis glicêmicos", ou seja, dosagens de glicemia de jejum e glicemias pós-prandiais, colhidas 2 horas após o café da manhã e 2 horas após o almoço das pacientes, em um mesmo dia. Além disso, era realizada uma medida da glicemia capilar no momento de cada consulta, que era utilizada exclusivamente para a análise de inconsistências na dieta ou nas medidas da glicemia. A realização dos perfis glicêmicos se dava com frequência semanal ou quinzenal, individualizada de acordo com o grau de controle de cada paciente. A partir de 2003, também foram determinadas a hemoglobina glicada (HbAlc) das pacientes, ao diagnóstico e mensalmente, para avaliação do nível e/ou tempo de hiperglicemia prévio ao diagnóstico, e a eventual correlação com a evolução do controle glicêmico nas pacientes de modo individual. Antes de 2003, a avaliação integrada do controle glicêmico era realizada por meio da dosagem de frutosamina. Como a amostra de pacientes analisadas até 2003 foi relativamente pequena ( 9 pacientes - $0,97 \%)$, a correlação da frutosamina com os valores glicêmicos não foi avaliada.

Todas as pacientes foram orientadas quanto ao tratamento dietético. $\mathrm{O}$ controle glicêmico foi considerado bom se: glicemia de jejum $\leq 95 \mathrm{mg} / \mathrm{dL}$ e glicemia pós-prandial $\leq 120 \mathrm{mg} / \mathrm{dL}$ (3). Quando, após duas semanas de dieta, os níveis glicêmicos permaneceram elevados - de jejum, maior ou igual a $105 \mathrm{mg} / \mathrm{dL}$ e de duas horas pós-prandiais, maior ou igual a $130 \mathrm{mg} / \mathrm{dL}$-, foi iniciado tratamento com insulina.

A avaliação estatística, teste t-Student e teste do qui-quadrado $\left(\chi^{2}\right)$ foram realizados por intermédio do programa Statistica 7.0, sendo considerado um nível de significância de $5 \%$.

\section{RESULTADOS}

As principais características das gestantes avaliadas neste estudo encontram-se na tabela 1.
Tabela 1. Características das gestantes com DMG (média \pm DP)

\begin{tabular}{lc}
\hline Características & Valor \\
\hline $\mathrm{N}$ (gestações) & 924 \\
Idade (anos) & $31,9 \pm 6,5$ \\
Idade gestacional na 1ª consulta (sem) & $31,0 \pm 5,1$ \\
Idade gestacional ao diagnóstico (sem) & $26,4 \pm 5,0$ \\
IMC pré-gestacional (kg/m²) & $27,8 \pm 5,9$ \\
IMC ao diagnóstico de DMG (kg/m²) & $31,2 \pm 5,7$ \\
Altura (cm) & $158,7 \pm 7,0$ \\
Glicemia de jejum rastreamento (mg/dL) & $92,8 \pm 30,6$ \\
Glicemia de jejum no TOTG (mg/dL) & $97,6 \pm 22,5$ \\
Glicemia 2h pós-prandial no TOTG (mg/dL) & $168,4 \pm 31,1$ \\
\hline
\end{tabular}

DMG: diabetes melito gestacional, TOTG: teste oral de tolerância à glicose.

\section{Fatores de risco e sua relação com o uso de insulina}

Quase todas as pacientes $(95,1 \%)$ apresentavam algum dos fatores de risco para diabetes melito gestacional, sendo que metade delas tinha três ou mais fatores associados (Tabela 2). Alguns fatores de risco, como a SOP, história de macrossomia e polidrâmnio em gestação anterior, nunca ocorreram como fatores de risco isolados. As pacientes que não possuíam fatores de risco para DMG tinham uma média de idade menor (21,6 $\pm 3,2$ anos) do que as com fatores de risco $(32,4 \pm 6,2$ anos $),(\mathrm{p}<0,001)$. Além disso, $14 \%$ delas necessitaram de tratamento com insulina e 18\% apresentaram algum tipo de complicação durante a gestação, o que não foi estatisticamente diferente quando comparado com o grupo das pacientes com fatores de risco $(\mathrm{p}=0,27 ; \mathrm{p}=0,47$, respectivamente $)$.

Tabela 2. Porcentagem de fatores de risco entre as pacientes com DMG

\begin{tabular}{ccc}
\hline $\begin{array}{c}\text { Quantidade de fatores } \\
\text { de risco }\end{array}$ & Pacientes $(\mathbf{n})$ & \% \\
\hline 0 & 45 & 4,87 \\
1 & 178 & 19,27 \\
2 & 235 & 25,43 \\
3 & 207 & 22,40 \\
4 & 150 & 16,23 \\
5 & 58 & 6,28 \\
6 & 34 & 3,68 \\
7 & 15 & 1,62 \\
9 & 2 & 0,22 \\
\hline
\end{tabular}

Como a maioria das pacientes tinha idade superior a 25 anos, avaliamos a porcentagem de pacientes com fatores de risco excluindo esse fator, e mesmo assim $85,6 \%$ das gestantes continuavam possuindo algum indicador de risco para DMG. Apenas 1,6\% das pacientes tinha idade inferior a 18 anos. Considerando-se a maior 
probabilidade de diabetes melito tipo 1 (DMl) neste grupo de pacientes mais jovens e visto que sua instalação pode coincidir ou ser acelerada pela gestação, da mesma forma que o DM2, as pacientes foram acompanhadas com atenção a essa possibilidade e orientadas quanto aos sintomas de insulinopenia mais intensa. Entretanto, a avaliação de autoimunidade contra as células beta-pancreáticas por meio da dosagem dos anticorpos contra células da ilhota ou o anti-GAD (a descarboxilase do ácido glutâmico) não foi realizada. Não foram constatadas diferenças em relação à época do diagnóstico $(26,4 \pm 5,0$ semanas versus $26,7 \pm 5,7$ semanas de idade gestacional; $\mathrm{p}=0,42$ ) ou na evolução, entre pacientes com idade superior ou inferior a 18 anos, comparando-se fatores como complicações durante a gestação em curso $(p>0,6)$ ou uso de insulina $(p=0,50)$.

A tabela 3 mostra os principais fatores de risco avaliados e suas respectivas porcentagens.

Tabela 3. Prevalência dos fatores de risco nas pacientes com DMG

\begin{tabular}{lc}
\hline Fatores de risco & Prevalência (\%) \\
\hline Idade superior a 25 anos & 83,1 \\
História familiar em parente de primeiro grau & 37,5 \\
Obesidade & 25,4 \\
Hipertensão arterial & 19,4 \\
Ganho de peso excessivo na gestação atual & 16,8 \\
Baixa estatura & 12,5 \\
DHEG na gestação atual & 10,7 \\
Óbito fetal ou abortos de repetição prévio & 10,1 \\
Macrossomia prévia & 10,3 \\
Diabetes melito gestacional prévio & 8,6 \\
Natimorto prévio & 4,3 \\
Malformação fetal prévia & 1,8 \\
Polidrâmnio prévio & 1,2 \\
\hline
\end{tabular}

DHEG: doença hipertensiva específica da gestação.

A presença de diabetes na família materna da paciente foi mais prevalente $(24,3 \%$ das pacientes) do que na família paterna [em $9,4 \%$ das pacientes $\left(\chi^{2}=52,4\right.$; $\mathrm{p}<0,001)]$, dentre as pacientes que tinham história de diabetes em parente de primeiro grau. Em 3,8\% das gestantes, ambos os pais tinham sido diagnosticados como portadores de diabetes. A presença de diabetes em familiar de segundo grau, classicamente não considerada como fator de risco para DMG, foi de $24,7 \%$ no presente estudo. O parente de segundo mais afetado nestes casos foi a avó materna ( $11,4 \%$ do total), seguida do irmão/irmã $(7,7 \%)$, avó paterna $(5,4 \%)$, avô materno $(2,5 \%)$ e, por fim, avô paterno $(2,0 \%)$. Em apenas 5 pacientes, a história de familiar de segundo grau com diabetes foi o único fator de risco encontrado.

Em relação a outro fator de risco muito frequente nessas pacientes, a obesidade, calculada com base no IMC pré-gestacional $\left(\geq 30 \mathrm{~kg} / \mathrm{m}^{2}\right)$, esteve presente em $25,4 \%$. A média do IMC pré-gestacional $\left(\mathrm{kg} / \mathrm{m}^{2}\right)$ foi de $27,8 \pm 5,9$, variando entre 15,3 e 55,5 . Já o IMC ao diagnóstico foi, em média, de $31,2 \pm 5,7$, sendo que essas pacientes tiveram um ganho de peso médio do início da gestação até o diagnóstico de $8,8 \mathrm{~kg}(0,33 \mathrm{~kg} / \mathrm{semana}$ em média). Cento e cinquenta e cinco pacientes (16,8\%) se enquadraram no critério de ganho de peso excessivo até o diagnóstico de DMG, definido como um ganho superior a $500 \mathrm{~g}$ por semana de gestação, que também é considerado um dos fatores de risco para DMG. Do total de pacientes, $115(15,4 \%)$ tiveram ganho de peso excessivo sem diagnóstico de obesidade, $32(4,3 \%)$ possuíam ambos os fatores de risco e $198(26,5 \%)$, somente obesidade. Na tabela 4 , podemos observar a correlação desses fatores com a média de ganho de peso até o diagnóstico de diabetes gestacional. Das pacientes com obesidade, $43,4 \%$ necessitaram usar insulina, contra $25,7 \%$ das que não tinham obesidade $\left(\chi^{2}=18,35 ; \mathrm{p}<0,001\right)$.

Tabela 4. Correlação entre os níveis de obesidade e ganho de peso excessivo até 0 diagnóstico de DMG

\begin{tabular}{|c|c|c|c|c|}
\hline & \multicolumn{2}{|c|}{$\begin{array}{c}\text { Ganho de peso } \\
\text { excessivo } \\
\text { até o diagnóstico }\end{array}$} & \multicolumn{2}{|c|}{$\begin{array}{c}\text { Sem ganho de peso } \\
\text { excessivo } \\
\text { até o diagnóstico }\end{array}$} \\
\hline & N médio* & $\begin{array}{l}\text { Ganho } \\
\text { de peso }\end{array}$ & N médio* & $\begin{array}{r}\text { Ganho } \\
\text { de peso }\end{array}$ \\
\hline Sem obesidade $(\mathrm{IMC} \leq 30)$ & $115(15,4 \%)$ & 17,27 & $496(53,7 \%)$ & 7,70 \\
\hline Obesidade (IMC $\geq 30$ ) & $32(4,3 \%)$ & 16,51 & $(6,5 \%)$ & 4,77 \\
\hline
\end{tabular}

* A média de ganho de peso é expressa em quilogramas $(\mathrm{kg})$; 0 critério de obesidade é baseado no IMC pré-gestacional (IMC $\geq 30 \mathrm{mg} / \mathrm{kg}^{2}$ ) e 0 ganho de peso excessivo é o superior a $500 \mathrm{~g} /$ semana de gestação.

Quando avaliamos a associação de fatores de risco, pode ser observado que pacientes com história familiar de diabetes em parente de primeiro grau e obesidade (11,9\% do total) frequentemente necessitaram de insulina, ou seja, $45,4 \%$ necessitaram de tratamento complementar à dieta, comparado a $18,2 \%$ das que não possuíam nenhum dos dois fatores de risco $\left(\chi^{2}=18,15, \mathrm{p}<0,005\right)$.

Das pacientes que tinham diagnóstico de diabetes gestacional prévio, um pouco mais da metade $(53,7 \%)$ necessitou usar insulina durante a gestação, contra $28,0 \%$ das que não tinham história de $\mathrm{DMG}$ prévio $\left(\chi^{2}\right.$ $=10,65 ; \mathrm{p}<0,005)$. As pacientes com DMG prévio também tiveram maiores médias de glicemia de jejum inicial $(108,7 \pm 39,8 ; \mathrm{p}<0,001)$, glicemia de jejum no TTOG $(109,1 \pm 28,3 ; \mathrm{p}<0,001)$ e média glicê- 
mica durante o acompanhamento $(111,7 \pm 25,9 ; \mathrm{p}<$ $0,001)$, além do diagnóstico mais precoce, com 23,6 \pm 6,5 semanas em média contra $26,6 \pm 4,8$ semanas nas pacientes sem DMG prévio $(\mathrm{p}<0,001)$.

Analisando-se a associação entre diabetes gestacional prévio e história familiar de diabetes em parente de primeiro grau, a prevalência e a porcentagem de necessidade de insulina nos grupos de pacientes que tinham ambos os fatores de risco, nenhum destes fatores de risco, apenas DMG prévio, e somente história familiar de diabetes, estão listadas na tabela 5 . Foi encontrada diferença significativa na comparação entre os grupos que tinham ambos os fatores de risco, história familiar de diabetes e DMG prévio, com uso de insulina, versus o grupo sem nenhum desses fatores e a necessidade de insulina, $70 \%$ versus $23,9 \%\left(\chi^{2}=14,35, \mathrm{p}<0,005\right)$.

Tabela 5. Prevalência e necessidade de insulina de gestantes entre grupos de pacientes com DMG prévio e história familiar de diabetes em parente de primeiro grau

\begin{tabular}{lcccccc}
\hline \multirow{2}{*}{ Fatores de risco } & \multicolumn{2}{c}{ Prevalência } & & \multicolumn{2}{c}{$\begin{array}{c}\text { Necessidade } \\
\text { de insulina }\end{array}$} \\
\cline { 2 - 3 } \cline { 6 - 6 } & $\mathbf{n}$ & $\mathbf{\%}$ & & $\mathbf{n}$ & $\mathbf{\%}$ \\
\hline DMG prévio & 50 & 5,4 & & 22 & 44 \\
$\begin{array}{l}\text { História familiar de diabetes } \\
\text { em parente de primeiro grau }\end{array}$ & 317 & 34,3 & & 110 & 35 \\
$\begin{array}{l}\text { DMG prévio + história } \\
\text { familiar de diabetes em } \\
\text { parente de primeiro grau }\end{array}$ & 30 & 3,2 & & 21 & 70 \\
\begin{tabular}{l} 
Nenhum desses fatores \\
\hline
\end{tabular} & 527 & 57 & & 125 & 23,3 \\
\hline
\end{tabular}

Diagnóstico, controle glicêmico e tratamento durante a gestação

A média da glicemia de jejum inicial $(\mathrm{mg} / \mathrm{dL})$ das pacientes, realizada como exame de rotina na primeira consulta de pré-natal e que serviu como rastreamento para o DMG, foi de 92,8 $\pm 30,6 \mathrm{mg} / \mathrm{dL}$, sendo que $54,1 \%$ dessas pacientes realizaram essa medida no primeiro trimestre de gestação $(12,6 \pm 5,6$ semanas em média). Desse grupo, 38,7\% tinham glicemia de jejum abaixo de $85 \mathrm{mg} / \mathrm{dL}$ e, destas, 17,3\% necessitaram de tratamento com insulina na evolução da gestação, em comparação a $38,8 \%$ das que tinham glicemia de jejum inicial $\geq 85\left(\chi^{2}=22,89 ; p<0,001\right)$.

$\mathrm{O}$ diagnóstico de $\mathrm{DMG}$ nas pacientes com glicemia de jejum $<85 \mathrm{mg} / \mathrm{dL}$ que necessitaram de insulina se deu em média na $27^{\text {a }}$ semana.

Se avaliarmos as pacientes com rastreamento negativo (glicemia de jejum no rastreamento $<85 \mathrm{mg} / \mathrm{dL}$ ) e que não possuíam fatores de risco, ou seja, para as quais alguns autores consideram que a investigação não seria obrigató- ria (3,0\% do total ou 28 pacientes), apenas 1 delas necessitou usar insulina durante o acompanhamento, porém 4 pacientes (14,3\% destas) apresentaram algum tipo de complicação na gestação estudada $(7,1 \%$ de polidrâmnio, $3,6 \%$ de malformação fetal e $10,7 \%$ de macrossomia).

Uma pequena porcentagem das pacientes, 92 pacientes $(9,9 \%)$, já possuía glicemia inicial acima de $110 \mathrm{mg} / \mathrm{dL}$, o que foi confirmado com uma segunda glicemia de jejum imediatamente ou como parte da realização do TOTG de $75 \mathrm{~g}$ em 86,9\% dos casos. A necessidade de tratamento além do dietoterápico nessas pacientes foi alta $(91,2 \%$ contra $19,3 \%$ nas pacientes que não tinham 2 glicemias de jejum acima de $\left.110 \mathrm{mg} / \mathrm{dL}, \chi^{2}=76,18 ; \mathrm{p}<0,001\right)$, e o uso de insulina também foi elevado nas pacientes que tinham glicemia de jejum inicial > 110 mesmo quando esse achado não foi confirmado com uma segunda glicemia de jejum, chegando a $72,7 \%$. Nesses casos, não pode ser descartada a realização de dieta para diabetes, entre as duas glicemias, ainda que não formalmente indicada. De qualquer modo, a segunda glicemia normal pode refletir uma anormalidade glicêmica menos intensa.

O diagnóstico de diabetes gestacional se deu, em média, por volta da $26^{\mathrm{a}}$ semana de gestação, sendo que a glicemia de jejum média $(\mathrm{mg} / \mathrm{dL})$ ao diagnóstico foi de $97,6 \pm 22,5$ e a glicemia $2 \mathrm{~h}$ após sobrecarga $(\mathrm{mg} /$ $\mathrm{dL}$ ) de $168,4 \pm 31,1$. A relação entre pontes de corte de valores de glicemia de jejum e $2 \mathrm{~h}$ após sobrecarga encontrados no TOTG e sua associação com o uso de insulina encontram-se na tabela 6.

A média glicêmica $(\mathrm{mg} / \mathrm{dL})$, médias de glicemia de jejum e pós-prandiais realizadas em laboratório, durante a gestação, avaliada em 898 gestantes, foi de $100,1 \pm$ 20,5 , variando entre 66,7 e 358,7 (as pacientes sem esses dados - 26 pacientes - foram analisadas com relação aos fatores de risco e demais informações disponíveis. A glicemia de jejum média $(\mathrm{mg} / \mathrm{dL})$ no acompanhamento dessas pacientes foi de $89,8 \pm 17,5$ e a glicemia pós-prandial média $(\mathrm{mg} / \mathrm{dL})$ das pacientes foi de $105,2 \pm 23,1$. Excluindo-se as pacientes com glicemia de jejum francamente alterada, ou com diagnóstico realizado no primeiro trimestre da gestação, nas quais a suspeita seria de diabetes pré-gestacional não identificado antes da gravidez, a média glicêmica (glicemia jejum e glicemias pós-prandiais) $(\mathrm{mg} / \mathrm{dL})$ durante a gestação foi de 98,2 $\pm 17,8$. Como já era esperada, a média glicêmica geral, a média de glicemia de jejum e a média de glicemia pós-prandial durante o acompanhamento foram maiores no grupo de pacientes que necessitaram 
de tratamento adicional à dieta (Tabela 7), inclusive porque foram determinantes da indicação de insulina.

Já a média da $\mathrm{HbAlc}$, avaliada em 757 pacientes, incluídas a partir de 2003 , foi de $5,55 \pm 0,77$, variando entre 3,6 e 11,4. A porcentagem de pacientes que possuía HbAlc acima do valor de referência (> 6\%) foi de $19,1 \%$, sendo que, destas, $67,6 \%$ necessitaram de tratamento além do dietético, contra $25,2 \%$ das pacientes que não tinham hemoglobina glicada alterada $\left(\chi^{2}=40,27, \mathrm{p}<0,001\right)$.

A necessidade de insulinoterapia durante a gestação ocorreu em 30,2\% das pacientes. Das que necessitaram receber insulina, o início das aplicações se deu em média na $29^{a}$ semana de gestação. Seis pacientes $(0,6 \%)$ fizeram uso de metformina após a publicação dos resultados do MiG Trial em maio de 2008 (14), que corroborou dados prévios de segurança de utilização de metformina durante a gestação. Em nosso estudo, a metformina foi indicada pelas seguintes razões: déficit visual e consequente dificuldade na aplicação de insulina - 1 paciente, obesidade moderada a mórbida -4 pacientes, e início de tratamento após 37 semanas de gestação - 1 paciente. Essas pacientes não apresentaram maior média glicêmica ou média de hemoglobina glicada do que as pacientes que não fizeram uso de metformina, porém em duas gestações foi observado crescimento fetal excessivo, após a introdução do antidiabético oral.

Das pacientes que iniciaram o acompanhamento com a Endocrinologia com 37 semanas ou mais de ges- tação, aproximadamente $20 \%$ necessitaram de tratamento além do dietoterápico contra $31 \%$ nas que iniciaram o pré-natal mais precocemente $(\mathrm{p}=0,08)$. Levando-se em consideração as complicações obstétricas na gestação analisada, por volta de $30 \%$ daquelas apresentaram alguma ocorrência contra $27 \%$ destas ( $\mathrm{p}=0,78)$.

\section{Complicações}

Em relação às complicações durante a gestação, 243 pacientes $(26,3 \%)$ apresentaram algum tipo de ocorrência, estando estas listadas na tabela 8 . As malformações fetais são listadas a seguir: braquicefalia -1 , pielectasia bilateral -1 , hidrocefalia -5 , hidronefrose com rins multicísticos e ausência de bexiga -3 , hidronefrose com megabexiga - 1, hidronefrose com malformação renal -2 , fenda palatina -1 , malformação cardíaca -3 , comunicação intraventricular (CIV) - 1, microcefalia e encefalocele - 1 . Apenas em 2 gestações em que foram encontradas malformações fetais a glicemia no rastreamento foi maior do que $110 \mathrm{mg} / \mathrm{dL}$ (uma com $118 \mathrm{e}$ outra com $226 \mathrm{mg} / \mathrm{dL}$, realizadas com 19 e 8 semanas de gestação, respectivamente), apontando para a existência de diabetes pré-gestacional e hiperglicemia por ocasião da concepção. A média de glicemia de jejum $(\mathrm{mg} / \mathrm{dL})$ no rastreamento entre o grupo de pacientes que tinham glicemia inicial $<110 \mathrm{mg} / \mathrm{dL}$ (18 pacientes) foi de 80,8 , variando de 66 a 97, sendo estas realizadas por volta da $11^{\text {a }}$ semana de gestação.

Tabela 6. Comparação entre grupos em relação à glicemia de jejum e 2h pós-sobrecarga no TOTG em relação à prevalência e ao uso de insulina

\begin{tabular}{|c|c|c|c|c|c|c|}
\hline & \multicolumn{3}{|c|}{ GJ no TOTG } & \multicolumn{3}{|c|}{ G2h TOTG } \\
\hline & GJ $\geq 95^{*}$ & GJ $<95^{*}$ & p valor & $\mathrm{G} 2 \mathrm{~h} \geq 200^{*}$ & G2h $<200^{*}$ & p valor \\
\hline \multicolumn{7}{|l|}{ Prevalência } \\
\hline$n$ & 428 & 491 & & 119 & 759 & \\
\hline$\%$ & 46,32 & 53,13 & & 12,9 & 82,1 & \\
\hline Necessidade de insulina & & & $<0,001$ & & & $<0,001$ \\
\hline$n$ & 220 & 53 & & 69 & 165 & \\
\hline$\%$ & 51,4 & 10,7 & & 58 & 21,7 & \\
\hline
\end{tabular}

GJ: glicemia de jejum; G2h: glicemia 2 h após sobrecarga com $75 \mathrm{~g}$ de glicose; TOTG: teste oral de tolerância à glicose. * 0 s valores das glicemias estão expressos em mg/dL.

Tabela 7. Comparação entre controle glicêmico das pacientes com dieta e das pacientes com insulina ou ADO1

\begin{tabular}{|c|c|c|c|c|c|}
\hline \multirow{2}{*}{ Controle glicêmico } & \multicolumn{2}{|c|}{ Dieta } & \multicolumn{2}{|c|}{ Insulina ou ADO } & \multirow{2}{*}{$\mathrm{p}$ valor } \\
\hline & Valor & $\mathbf{n}$ & Valor & $\mathbf{n}$ & \\
\hline Glicemia média (mg/dL) & $92,4 \pm 9,2$ & 620 & $117,2 \pm 27,4$ & 278 & $<0,0001$ \\
\hline Glicemia de jejum média (mg/dL) & $83,4 \pm 9,3$ & 619 & $103,9 \pm 22,4$ & 277 & $<0,0001$ \\
\hline Glicemia pós-prandial média (mg/dL) & $97,0 \pm 11,3$ & 614 & $123,3 \pm 31,0$ & 276 & $<0,0001$ \\
\hline Hemoglobina glicada média (\%) & $5,35 \pm 0,53$ & 506 & $5,96 \pm 0,99$ & 252 & $<0,0001$ \\
\hline
\end{tabular}

ADO: antidiabético oral; diferenças estatísticas são mostradas por $p<0,05$. 
Tabela 8. Complicações obstétricas na gestação atual

\begin{tabular}{lc}
\hline Ocorrência & Prevalência \\
\hline DHEG & $10,7 \%$ \\
Macrossomia & $9,6 \%$ \\
Polidrâmnio & $6,6 \%$ \\
Malformação fetal & $2,2 \%$ \\
Sofrimento fetal agudo & $1,3 \%$ \\
Óbito fetal & $1,1 \%$ \\
\hline
\end{tabular}

\section{DISCUSSÃO}

A porcentagem de pacientes que possuíam fatores de risco para DMG na nossa população foi elevada, como igualmente observado em outros estudos $(15,16)$. Entretanto, a presença desses fatores de risco também tem sido encontrada em grande parte das gestantes que não evoluem para DMG, podendo estar presente em 20\% a $90 \%$ das gestantes de uma forma geral (16-18). Por outro lado, reconhece-se que a pesquisa de fatores de risco pós-diagnóstico de DMG é realizada de modo mais efetivo.

Em nosso estudo, apenas 3\% das pacientes com DMG não teriam sido investigadas se tivessem sido adotados os critérios de rastreamento seletivo recomendados pela American Diabetes Association (ADA)

A história familiar de diabetes tipo 2 é considerada um forte fator de risco para DMG, com relato de aumento de $68 \%$ no risco para a doença na presença desse fator (19). Além disso, diversos estudos têm demonstrado uma grande prevalência de diabetes materno em pacientes com diabetes tipo 2 em diferentes populações $(20,21)$. Bo e cols., analisando a prevalência de história familiar de diabetes em pacientes com diabetes tipo 2, encontraram uma prevalência de diabetes materna, paterna e em outros familiares, de $25,5 \%, 6,5 \%$ e $21,2 \%$, respectivamente. A porcentagem de diabetes em familiar de primeiro grau em geral e de diabetes materno encontrada nas pacientes com DMG no presente estudo não foi significativamente diferente da encontrada por Bo e cols. ( $\mathrm{p}=0,38 ; \mathrm{p}=0,58$, respectivamente), (Tabela 9), o que pode reforçar a estreita relação entre o DMG e o DM2. A influência da presença de diabetes nos pais tem sido associada na literatura com início mais precoce de DM2 e níveis de LDL-colesterol maiores do que em pacientes sem história parental de diabetes. Ao contrário, a presença de diabetes em familiares, excluindo os pais, parece estar relacionada apenas a um pequeno risco de início mais precoce de diabetes tipo 2 (21). $\mathrm{Na}$ população com diabetes gestacional, ainda não existem muitos estudos correlacionando os diferentes tipos de herança familiar com a evolução glicêmica. Em nosso estudo, a idade média $(\mathrm{p}>0,5)$ e a época do diagnóstico do DMG $(\mathrm{p}>0,1)$ não foram diferentes entre as pacientes com e sem história familiar de diabetes; o perfil lipídico não foi estudado.

Já a obesidade, outro fator de risco muito prevalente no nosso estudo, é considerada o maior fator de risco modificável relacionado ao diabetes gestacional. Uma recente metanálise concluiu haver probabilidade quase quatro vezes maior de desenvolvimento de DMG em pacientes obesas (IMC > 30) do que em pacientes de peso normal (22) e sugeriu que, para cada aumento de 1 $\mathrm{kg} / \mathrm{m}^{2}$ no IMC, a prevalência de DMG aumentaria em $0,92 \%$. Uma grande limitação da maioria dos estudos é a avaliação do ganho de peso total durante a gestação

Tabela 9. Comparação entre prevalência de DM em familiares de pacientes com diabetes gestacional em nosso estudo e em pacientes com DM2, de Bo e cols.

\begin{tabular}{|c|c|c|c|c|c|}
\hline \multirow{2}{*}{ História familiar de DM } & \multicolumn{2}{|c|}{ Em nosso estudo } & \multicolumn{2}{|c|}{ Bo e cols. } & \multirow{2}{*}{ p valor } \\
\hline & n (924) & $\%$ & n (2113) & $\%$ & \\
\hline Parente de primeiro grau & 347 & 37,55 & 677 & 32,03 & 0,38 \\
\hline Mãe & 225 & 24,35 & 540 & 25,55 & 0,58 \\
\hline Pai & 87 & 9,41 & 137 & 6,48 & $0,008^{*}$ \\
\hline Ambos os pais & 35 & 3,78 & & & \\
\hline Parente de segundo grau & 266 & 24,67 & 447 & 21,2 & $0,004^{*}$ \\
\hline Avó materna & 103 & 11,14 & & & \\
\hline Irmão & 71 & 7,68 & & & \\
\hline Avó paterna & 50 & 5,41 & & & \\
\hline Avô materno & 23 & 2,48 & & & \\
\hline Avô paterno & 19 & 2,05 & & & \\
\hline
\end{tabular}

Diferenças estatísticas são mostradas por ${ }^{*}, p<0,05$. 
e não a análise até o diagnóstico de DMG. Estudos demonstram que o ganho de peso no primeiro trimestre é significantemente maior no grupo de pacientes com DMG comparadas a gestantes controles $(3,40$ vs. 1,87 $\mathrm{kg}$ ) (23). Uma comparação na literatura entre pacientes com DMG e controles em relação ao ganho de peso até o screening realizado entre a $24^{\mathrm{a}}$ e $28^{\mathrm{a}}$ semana foi em média de $0,34 \mathrm{~kg} / \mathrm{semana}$ em ambos os grupos, porém com ganho de peso maior no primeiro trimestre para pacientes com DMG $(0,25 \mathrm{~kg} / \mathrm{sem}$ versus 0,17 $\mathrm{kg} /$ semana) (24). Em nosso estudo, o ganho de peso médio até o diagnóstico de DMG foi de $8,8 \mathrm{~kg}(0,33$ $\mathrm{kg} / \mathrm{sem})$. A obesidade não apenas foi prevalente como foi também um fator preditivo para a necessidade de insulina. Dessa forma, a prevenção da obesidade nas mulheres em idade reprodutiva e o cuidado em relação ao ganho de peso principalmente durante o primeiro trimestre potencialmente reduziriam não apenas a prevalência, como também a gravidade do DMG.

Estudos recentes têm demonstrado que, além dos fatores de risco clássicos pra $\mathrm{DMG}$, uma história de SOP também pode estar associada a um aumento no risco para DMG (25). Existem, porém, muitas controvérsias em relação a essa associação, pois em estudos casos controle ela não foi encontrada (26). No presente estudo, esse fator não foi frequentemente referido pelas pacientes ou em seu prontuário médico, que inclui a avaliação das demais clínicas do hospital, mas não foram realizados exames específicos para seu diagnóstico. Uma glicemia de jejum inicial abaixo de 85 $\mathrm{mg} / \mathrm{dL}$, realizada como rastreamento, na ausência de fatores de risco para diabetes gestacional, foi frequentemente associada com uma boa evolução durante a gestação. Entretanto, 1 paciente $(3,6 \%)$, de um total de 28 pacientes, sem fatores de risco para DMG e com glicemia de jejum inicial abaixo de $85 \mathrm{mg} / \mathrm{dL}$, necessitou usar insulina durante a gestação, e 4 pacientes (14,3\% destas) apresentaram algum tipo de complicação durante esse período, que incluíram: polidrâmnio, malformação fetal e crescimento fetal excessivo. De qualquer modo, a sistematização na pesquisa de fatores de risco para DMG, entre os inúmeros dados a serem pesquisados pelos obstetras, é um fator crucial para o sucesso de abordagens centradas nos fatores de risco. Tendo-se em mente que em países em desenvolvimento a gestação é um período ímpar para o diagnóstico e prevenção de inúmeras doenças, entre elas o diabetes melito, pode não ser recomendável dispensar o teste diagnóstico.
Uma questão que recentemente vem sendo debatida é o valor da HbAlc no diagnóstico do DMG, porém até o momento existem muitas dúvidas em relação a esse assunto, sendo considerada controversa a utilização da $\mathrm{HbAl} c$ até mesmo como parâmetro de avaliação de controle glicêmico durante a gestação. Sua utilização, entretanto, poderia ser uma estratégia interessante para o diagnóstico, uma vez que o TOTG requer o jejum das pacientes, é dispendioso, caro, desconfortável em função das náuseas e vômitos frequentes durante a gestação, além de ser pouco reprodutível. Um estudo avaliou a média de $\mathrm{HbAlc}$ em uma população de gestantes com DMG e obteve como resultado um valor de $6,00 \pm 0,81$, comparado à média da população gestante com tolerância normal à glicose, que ficou em 5,36 \pm $0,36(27)$. Esse valor não foi diferente da média encontrada em nossa amostra $(5,55, \pm 0,77)$, com $19,15 \%$ dessas pacientes possuindo média de $\mathrm{HbAl}$ c maior do que 6\% (limite superior de normalidade para o ensaio), e uma grande porcentagem destas necessitando de insulina. O menor valor de $\mathrm{HbAl}$ c média encontrado em pacientes que posteriormente necessitaram de insulina foi de 4,2\%. Esses achados entram em contraste com Aldasouqui e cols., que encontraram valores de hemoglobina glicada acima de $6 \%$ em até $87,1 \%$ das gestantes com DMG (28). Entre as pacientes do nosso estudo que tinham a primeira hemoglobina glicada medida < $5,5 \%, 20,5 \%$ precisaram de insulina durante a evolução da gestação e, das que tinham HbAlc $\geq 5,5 \%, 44,1 \%$ necessitaram usar insulina. Apesar da diferença significativa entre os dois grupos, não podemos correlacionar o valor da hemoglobina glicada com a necessidade sistemática de insulina como tratamento, pois houve uma grande porcentagem de pacientes no grupo de HbAlc $<5,5 \%$ que necessitaram de tratamento adicional (73 pacientes). Por isso, podemos afirmar apenas que a hemoglobina glicada não foi fator para início da insulinoterapia em nossas pacientes, porém, quando acima de 6\%, foi preditora da sua necessidade. Estudos controlados se fazem necessários para a validação dessa possível ferramenta diagnóstica adicional. A grande maioria das pacientes $(69,1 \%)$ conseguiu um bom controle glicêmico com medidas dietéticas, o que não foi diferente dos dados encontrados na literatura, que descreve o tratamento dietético como suficiente em $40 \%$ a $70 \%$ das gestantes com DMG (29). Observamos durante essa avaliação que as pacientes chegaram tarde para acompanhamento especializado no pré-natal, em média 5 semanas após a realização do diagnóstico de 
diabetes gestacional. Avaliando as pacientes que iniciaram o acompanhamento com a Endocrinologia após a $36^{\text {a }}$ semana de gestação, observamos que cerca de $20 \%$ necessitaram de tratamento além do dietoterápico e quase $30 \%$ apresentaram alguma complicação obstétrica na gestação estudada, provavelmente significando que essas pacientes foram encaminhadas pela deterioração do controle. Considerando-se que o diagnóstico do DMG é preconizado entre 24-28s, recomendamos estratégias que possam garantir o início mais precoce do seu manejo, como a localização da paciente e implementação de protocolos que permitam a instituição imediata do tratamento nas unidades de tratamento primário ou secundário, bem como o encaminhamento a unidades terciárias.

A porcentagem de malformações fetais encontrada em nossa amostra foi de $2,2 \%$ de todas as gestações e incluiu as seguintes anomalias: micro e braquicefalia, anomalias do sistema geniturinário, fenda palatina, malformações cardíacas, e encefalocele. Poucos estudos na literatura demonstram uma relação evidente entre essas malformações e o DMG, sendo descrito em um deles uma taxa de 2,8\% das gestações com DMG, em comparação com até $2,4 \%$ da população em geral (30). Essa correlação supostamente evidencia descontrole glicêmico no período da organogênese $(31,32)$. Apenas uma (1) das gestantes com malformação fetal em nosso estudo apresentou hiperglicemia franca ao diagnóstico (glicemia de jejum no rastreamento de $226 \mathrm{mg} / \mathrm{dL}$ ), realizado ainda no primeiro trimestre de gestação, o que poderia corresponder a um diabetes pré-gestacional e justificar sua ocorrência.

Podemos concluir que os fatores de risco utilizados neste estudo e na rotina do Serviço de Obstetrícia da UFPR são altamente sensíveis para o diagnóstico, visto que $95,1 \%$ das pacientes com DMG apresentavam um ou mais desses fatores, pesquisados após o diagnóstico da doença. Por outro lado, ainda que altamente sensíveis para o diagnóstico, reconhece-se que esses fatores são largamente inespecíficos, visto estarem presentes com frequência na população geral. A distribuição da história familiar segue o padrão observado fora da gestação para o DM2, sugerindo tratar-se da mesma doença, transitória ou precocemente revelada em função do efeito diabetogênico da gestação. Os dados reforçam a necessidade de atenção especial para pacientes com glicemia de jejum no rastreamento $\geq 85 \mathrm{mg} / \mathrm{dL}$, glicemia de jejum no TOTG $\geq 95 \mathrm{mg} / \mathrm{dL}$ e glicemia após $2 \mathrm{~h}$ de sobrecarga com $75 \mathrm{~g}$ de glicose $\geq 200 \mathrm{mg} / \mathrm{dL}$, DMG prévio, obesidade, HbAlc acima de 6\% e associação dos fatores de risco, história familiar de diabetes com obesidade ou DMG prévio, pois todos esses fatores foram preditivos de piora no controle glicêmico e uso de insulina no nosso estudo. O número de pacientes com glicemia de jejum inicial $<85 \mathrm{mg} / \mathrm{dl}$ na ausência de fatores de risco $(3,03 \%$ das pacientes) foi pequeno para permitir uma análise mais aprofundada, porém foi encontrado $7,1 \%$ de polidrâmnio, 3,6\% de malformação fetal e $10,7 \%$ de macrossomia neste grupo de pacientes e mesmo a necessidade de insulina em um dos casos. Em gestantes não diabéticas, a prevalência dessas complicações é de até $2,4 \%$ para malformação fetal em geral e $12 \%$ para macrossomia fetal $(30,32)$.

A hemoglobina glicada, cuja utilização não está formalmente indicada durante a gestação, não mostrou boa correlação com a necessidade de insulina, porém, como citado anteriormente, quando em nível superior a $6 \%$ foi fator preditor para seu uso.

Em nosso artigo, o fator mais determinante para a necessidade de insulina foi a associação entre DMG prévio e história familiar de diabetes em parente de primeiro grau, sendo então de fundamental importância que esses dados sejam sempre pesquisados no início do pré-natal.

Agradecimentos: agradecemos o apoio técnico na parte estatística da professora Vânia Manfredini de Alcântara (professora do curso de Farmácia e do curso de Pós-graduação de Ciências Farmacêuticas da Universidade Federal do Paraná).

Declaração: os autores declaram não haver conflitos de interesse científico neste estudo.

\section{REFERÊNCIAS}

1. American Diabetes Association. Diagnosis and classification of diabetes mellitus. Diabetes Care. 2006;29(1):S43-8.

2. The Expert Committee on the Diagnosis and Classification of Diabetes Mellitus. Report of the Expert Committee on the Diagnosis and Classification of Diabetes Mellitus. Diabetes Care. 1997;20:1183-97.

3. American Diabetes Association. Clinical Practice Recommendations. Gestational diabetes mellitus. Diabetes Care. 2004;27(1):S88-90.

4. Schmidt MI, Matos MC, Reichelt AJ, Forti AC, de Lima L, Duncan BB. Prevalence of gestational diabetes mellitus do the WHO criteria make a difference? Brazilian Gestational Diabetes Group. Diabet Medic. 2000;17:376-80.

5. Hillier TA, Vesco KK, Pedula KL, Beil TL, Whitlock EP, Pettitt DJ. Screening for gestational diabetes mellitus: a systematic review for the U.S. Preventive Services Task Force. Ann Intern Med. 2008;148:766-75.

6. Branchtein L, Schmidt MI, Matos MC, Yamashita T, Pousada JM, Duncan BB. Short stature and gestational diabetes in Bra- 
zil. Brazilian Gestational Diabetes Study Group. Diabetologia. 2000;43:848-51.

7. Anastasiou E, Alevizaki M, Grigorakis SJ, Philippou G, Kyprianou M, Souvatzoglou A. Decreased stature in gestational diabetes mellitus. Diabetologia. 1998;41:997-1001.

8. Reichelt AJ, Spichler ER, Branchtein L, Nucci LB, Franco LJ, Schmidt Ml; for the Brazilian Study of Gestational Diabetes (EBDG) Working Group: fasting plasma glucose is a useful test for the detection of gestational diabetes. Diabetes Care. 1998;21:1246-9.

9. Reichelt AJ, Oppermann MLR, Schimdt MI. Recomendações da $2^{\mathrm{a}}$ reunião de grupo de trabalho em diabetes e gravidez. Arq Bras Endocrinol Metab. 2002;46:574-81.

10. American Diabetes Association. Summary and Recommendations of the Fifth International Workshop-Conference on Gestational Diabetes Mellitus. Diabetes Care. 2007;30(2):S251-260.

11. World Health Organization. Definition, diagnosis and classification of diabetes mellitus and its complications: report of a WHO consultation. Geneva, WHO; 1999.

12. Hanson U, Björklund A, Hjertberg R, Eva N, Nordlander E. Maternal and fetal outcomes if gestational impaired glucose tolerance is not treated. Diabetes Care. 2003;26(7):2107-11.

13. Diabetes Prevention Program Research Group. Reduction in the Incidence of Type 2 Diabetes with Lifestyle Intervention or Metformin. N Engl J Med. 2002;346(6):393-403.

14. Rowan JA, Hague WM, Gao W, Battin MR, Moore MP; MiG Trial Investigators. Metformin versus insulin for the treatment of gestational diabetes. N Engl J Med. 2008;358:2003-15.

15. Cypryk K, Szymczak W, Czupryniak L, et al. Gestational diabetes mellitus - an analysis of risk factors. Pol $\mathrm{J}$ Endocrinol. 2008;59:393-7.

16. Williams $\mathrm{CB}$, Iqbal $\mathrm{S}$, Zawacki $\mathrm{CM}, \mathrm{Yu} \mathrm{D}$, Brown MB, Hernan WH. Effect of selective screening for gestational diabetes. Diabetes Care. 1999;22:418-21.

17. Nasrat HA, Ardawi MS, Arbalkhail BA. The diagnosis of "pathological hyperglycemia" in gestational diabetes in a high risk obstetric population. Diabet Med. 1996;13:861-7.

18. Weeks JW, Major CA, de Veciana M, Morgan MA. Gestational diabetes: does the presence of risk factors influence perinatal outcomes? Am J Obstet Gynecol. 1994;171:1003-7.

19. Solomon CJ, Willett WC, Carey VJ. A prospective study of pregravid determinations of gestational diabetes mellitus. JAMA. 1997;278:1078-83.
20. Papazafiropoulou A, Sotiropoulos A, Skliros E, Kardara M, Kokolaki A, Apostolou O. Familial history of diabetes and clinical characteristics in Greek subjects with type 2 diabetes. BMC Endocrine Disorders. 2009;9:12.

21. Bo S, Cavallo-Perin P, Gentile L, Repetti E, Pagano G. Influence of a familial history of diabetes on the clinical characteristics of patients with type 2 diabetes mellitus. Diabet Med. 2000;17:538-42.

22. Torloni MR, Betrán AP, Horta BL, Nakamura MU, Atallah AN, Moron $A F$, et al. Prepregnancy $B M I$ and the risk of gestational diabetes: a systematic review of the literature with meta-analysis. Obes Rev. 2009;10:194-203.

23. Morisset AS, Tchernof A, Dubé MC, Veillette J, Weisnagel SJ, Robitaille J. Weight gain measures in women with gestational diabetes mellitus. J Womens Health. 2011;20(3):375-80.

24. Heddderson M, Gunderson EP, Ferrara A. Gestational weight gain and risk of gestational diabetes mellitus. Obstet Gynecol. 2010;115(3):597-604.

25. Boomsma CM, Eijkemans MJC, Eijkemans EG, Visser GHA, Fauser BCJM, Macklon NS. A meta-analysis of pregnancy outcomes in women with polycystic ovary syndrome. Hum Reprod Update. 2006;16:673-83.

26. Toulis KA, Goulis DG, Kolibianakis EM, Venetis CA, Tarlatzis BC, Papadimas I. Risk of gestational diabetes mellitus in women with polycystic ovary syndrome: a systematic review and a meta-analysis. Fertil Steril. 2009;92:667-77.

27. Balaji V, Maduhri BS, Ashalatha S, Sheela S, Suresh S, Seshiah V. A1C in gestational diabetes in Asian Indian women. Diabetes Care. 2007;30:1865-7.

28. Aldasouqui SA, Solomon DJ, Bokhari DJ, Khan PM, Muneera S, Gossain VV. Glycohemoglobin A1c: a promising screening tool in gestational diabetes mellitus. Int J Diab Dev Ctries. 2008;28:121-4.

29. World Health Organization. Definition, diagnosis and classification of diabetes mellitus and its complications: report of a WHO consultation. Geneva, WHO; 1999.

30. Janssen PA, Rothman I, Schwartz SM. Congenital malformations in newborns of women with established and gestational diabetes in Washington State. Paediatr Perinat Epidemiol. 1996;10:52-63.

31. FarrelT, Neale L, CundyT. Congenital anomalies in the offspring of women with type1, type 2 and gestational diabetes. Diabet Med. 2002;19(4):322-6.

32. Cunningham FG, Gant NF, Leveno KJ, Gilstrap LC, Hauth JC, Wenstrom KD, editors. Williams obstetrics. 21st ed. New York, NY: McGraw-Hill; 2001. 